\title{
Molecular plasma deposition: biologically inspired nanohydroxyapatite coatings on anodized nanotubular titanium for improving osteoblast density
}

\author{
This article was published in the following Dove Press journal: \\ International Journal of Nanomedicine \\ 12 January 2015 \\ Number of times this article has been viewed
}

\author{
Ganesan Balasundaram' \\ Daniel M Storey' \\ Thomas J Webster ${ }^{2,3}$ \\ 'Chameleon Scientific, Longmont, \\ CO, USA; ${ }^{2}$ Department of Chemical \\ Engineering, Northeastern \\ University, Boston, MA, USA; ${ }^{3}$ Center \\ of Excellence for Advanced Materials \\ Research, King Abdulaziz University, \\ Jeddah, Saudi Arabia
}

\begin{abstract}
In order to begin to prepare a novel orthopedic implant that mimics the natural bone environment, the objective of this in vitro study was to synthesize nanocrystalline hydroxyapatite (NHA) and coat it on titanium (Ti) using molecular plasma deposition (MPD). NHA was synthesized through a wet chemical process followed by a hydrothermal treatment. NHA and micron sized hydroxyapatite (MHA) were prepared by processing NHA coatings at $500^{\circ} \mathrm{C}$ and $900^{\circ} \mathrm{C}$, respectively. The coatings were characterized before and after sintering using scanning electron microscopy, atomic force microscopy, and X-ray diffraction. The results revealed that the post-MPD heat treatment of up to $500^{\circ} \mathrm{C}$ effectively restored the structural and topographical integrity of NHA. In order to determine the in vitro biological responses of the MPD-coated surfaces, the attachment and spreading of osteoblasts (bone-forming cells) on the uncoated, NHA-coated, and MHA-coated anodized Ti were investigated. Most importantly, the NHA-coated substrates supported a larger number of adherent cells than the MHA-coated and uncoated substrates. The morphology of these cells was assessed by scanning electron microscopy and the observed shapes were different for each substrate type. The present results are the first reports using MPD in the framework of hydroxyapatite coatings on Ti to enhance osteoblast responses and encourage further studies on MPD-based hydroxyapatite coatings on Ti for improved orthopedic applications.
\end{abstract}

Keywords: hydroxyapatite, anodization, nanotechnology

\section{Introduction}

Over the past few decades, the field of biomaterials has shifted in emphasis from achieving a bioinert tissue response to stimulating specific cellular responses at the molecular level. Designing biomaterial surfaces to direct specific cellular responses in a predictable manner has drawn enormous attention; yet, little work has been done for one of our most common biomaterials: titanium (Ti) and Ti alloys. ${ }^{1-8}$ To solve these problems, surface modification seems to be a more economical and efficient way to promote immediate and long-term implant fixation (rather than using pharmaceutical agents, which may have side effects) thus avoiding long-term implant problems. ${ }^{1-8}$

Various surface modification techniques, such as chemical etching, electrochemical treatment, ion implantation, electron beam irradiation, and the application of a variety of coatings have been used to improve the function of Ti implants. ${ }^{1-8}$ Among them, the application of electrochemical oxidation has attracted increasing interest because of its simplicity, low cost, and controllability at the nanoscale. Electrochemical anodic oxidation could be used to grow a thick and uniform oxide layer on metals and several
Correspondence: Thomas J Webster Department of Chemical Engineering, Northeastern University, 360 Huntington Avenue, Boston, MA 02II5, USA

Tel + I 6173736585

Email th.webster@neu.edu 
alloys, such as Ti, aluminum, tantalum, and their alloys, and has been reported to form surface nanotubular structures. ${ }^{6-8}$

Another approach to improve fixation between hard tissue and Ti implants, and therefore increase implant lifetime, is to coat the metal surface with a bioactive material that can promote rapid bonding to natural bone. Among the various surface treatments that have been attempted, coatings with hydroxyapatite $(\mathrm{HA})\left(\mathrm{Ca}_{10}\left[\mathrm{PO}_{4}\right]_{6}[\mathrm{OH}]_{2}\right)$ have attracted particular attention over the last decade because HA has a similar chemical composition and structure to the mineral phase of human bone; nanocrystalline HA (NHA) better mimics the natural structure and chemistry of bone than micron crystalline HA (MHA). ${ }^{9-15}$ The beneficial effect of HA coatings on $\mathrm{Ti}$, such as stronger bonding between the bone and the implant, increases uniform bone ingrowth at the bone-implant interface and decreases the release of metal ions from the implant to the body. ${ }^{16-18}$ To date, a popular manner by which to coat Ti with HA has been through a high heat process called plasma spray deposition, which significantly increases HA crystal growth into the nonbiologically-inspired micron regime. ${ }^{19-25}$

Here, for the first time, we report a new coating method called molecular plasma deposition (MPD) to produce HA coatings with different crystallinity and particle size on $\mathrm{Ti}$. To promote bonding strength between the HA and an underlying Ti substrate, Ti was anodized as mentioned above to possess novel nanotubes since that would increase contact area and penetration of HA into Ti. Samples were characterized for material properties and cytocompatibility properties with osteoblasts. Results showed significant promise for the study of MPD as an improved method to coat Ti with NHA for enhancing numerous orthopedic applications.

\section{Materials and methods Preparation of NHA particles}

NHA particles were synthesized by a well-established wet chemical process followed by hydrothermal treatment (Figure 1A). ${ }^{26-28}$ Briefly, concentrated ammonium hydroxide was used to maintain a $\mathrm{pH}$ of 10 throughout the reaction. Ammonium phosphate at $0.6 \mathrm{M}$ and $1.0 \mathrm{M}$ calcium nitrate were slowly added to serve as the basis for phosphorous and calcium in the HA (at $3.6 \mathrm{~mL} / \mathrm{min}$ ), respectively. Calcium phosphate precipitation occurred while stirring for 10 minutes at room temperature. After 10 minutes, the suspension was centrifuged (at 1,000 rpm) and pelleted. For hydrothermal treatment, the HA precipitated aqueous solution was added to a $125 \mathrm{~mL}$ Teflon liner (Parr Instrument). The Teflon liner was sealed tightly in an autoclave (Parr Acid Digestion
Bombs 4748; Parr Instrument) and subjected to hydrothermal treatment at $120^{\circ} \mathrm{C}$ for 20 hours. After the hydrothermal treatment, the HA particles were rinsed three times with distilled water and then the precipitate was dried in an oven for 12 hours at $70^{\circ} \mathrm{C}$. The dried NHA powder was then crushed with a mortar and pestle for later use.

\section{NHA characterization}

All NHA powders were characterized by X-ray diffraction (XRD) using Cu-Ka radiation (Siemens D500 Kristalloflex; Bruker AXS Inc.), scanning electron microscopy (SEM) (JEOL JSM-35CF), and inductively coupled plasma-atomic emission spectroscopy conducted by Impact Analytical (Midland, MI, USA) to determine its $\mathrm{Ca} / \mathrm{P}$ ratio. A BET surface area analyzer (SA3100; Beckman Coulter) was used to measure the surface area of the particles, and the equivalent spherical crystallite size was then determined using a theoretical HA density of $3.16 \mathrm{~g} / \mathrm{cm}^{3}$. Transmission electron microscopy (JEOL 2000FX) was also used to characterize the geometry of the NHA particles.

\section{Anodized Ti preparation and characterization}

An anodization process was used to form nanotubes on $\mathrm{Ti}$ coupons. ${ }^{29-34}$ First, square $\left(1 \times 1 \mathrm{~cm}^{2}\right)$ Ti foils (Alfa Aesar) (thickness of $250 \mu \mathrm{m}$; 99.7\%) were cleaned ultrasonically with ethanol and water prior to anodization. The cleaned substrates were etched by soaking in a mixture of $5 \mathrm{M}$ nitric acid (Sigma-Aldrich Co., St Louis, MO, USA) and hydrofluoric acid (Sigma-Aldrich). Samples were then rinsed several times with deionized water. Then, the etched Ti samples were anodized in a $1.5 \%$ hydrofluoric acid solution. Using a direct-current power supply (Tekpower 3645A, USA), $20 \mathrm{~V}$ were applied for 10 minutes. The anode and cathode were kept parallel, with a separation distance of about $1 \mathrm{~cm}$ during anodization. Lastly, the Ti samples were rinsed thoroughly with deionized water and dried with nitrogen gas.

SEM measurements were then conducted to confirm the expected nanotubular surface features on the anodized $\mathrm{Ti}$ substrates. For this, samples were sputter-coated with a thin layer of gold using an Ernest Fullam Sputter Coater (Model AMS-76M) in a 100 mTorr vacuum in argon for 3 minutes at $10 \mathrm{~mA}$. SEM mages were obtained using a TESCAN MIRA/LSM at a $20 \mathrm{kV}$ accelerating voltage, and images were recorded using TESCAN-MIRA software.

Atomic force microscopy (AFM) interfaced with Nanoscope III imaging software was used to quantify surface roughness of the samples of interest. A scan rate of $2 \mathrm{~Hz}$ was used at selected 

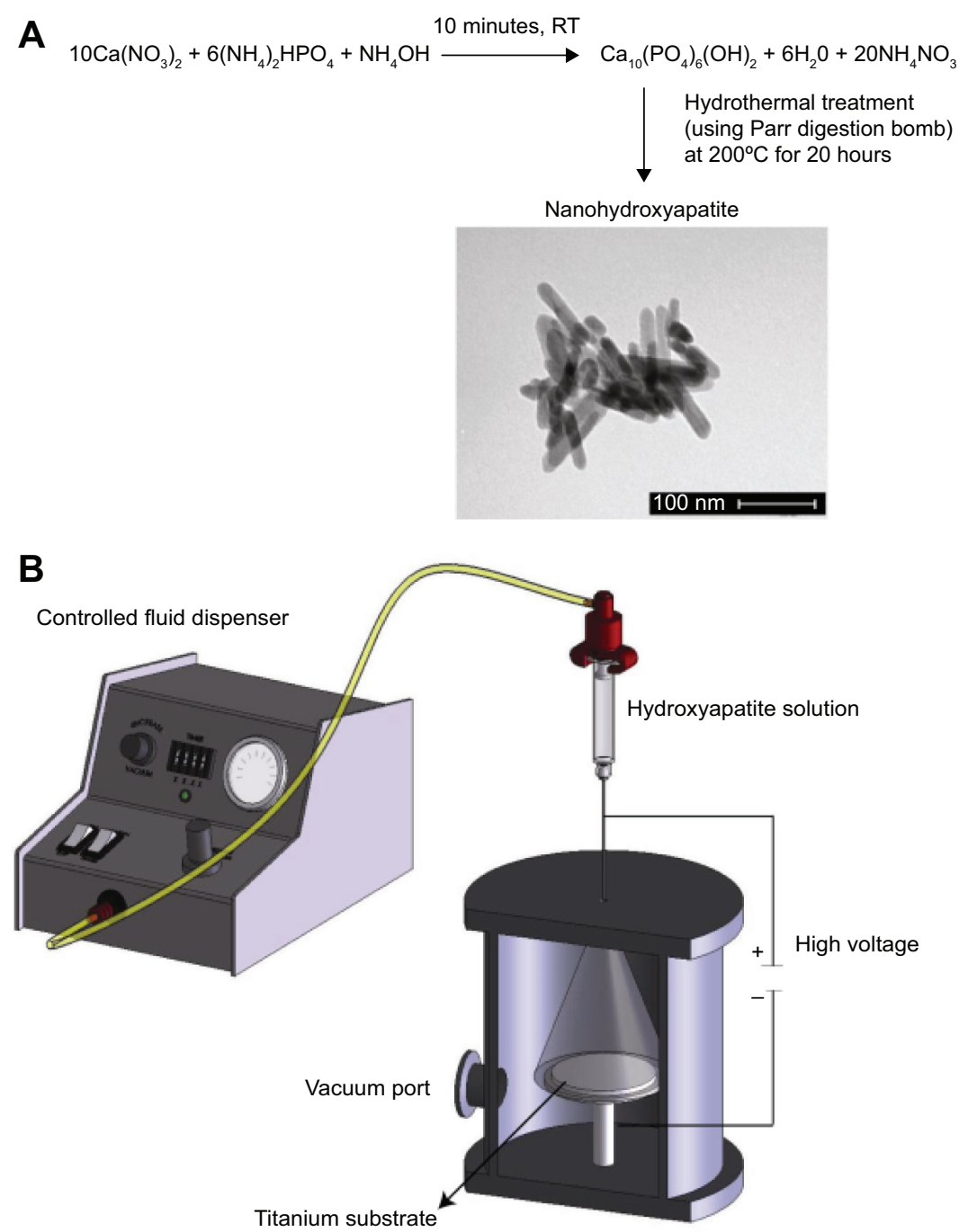

Figure I The NHA chemical synthetic scheme via hydrothermal treatment and TEM images of the synthesized NHA (A) and a sketch of the HA coating on anodized Ti using the novel MPD process (B).

Note: Scale bar $=100 \mathrm{~nm}$ for TEM image.

Abbreviations: HA, hydroxyapatite; MPD, molecular plasma deposition; NHA, nanocrystalline hydroxyapatite; RT, room temperature; TEM, transmission electron microscopy; $\mathrm{Ti}$, titanium.

scanning points to determine root mean square roughness values. AFM analysis (scan areas ranging from $1 \times 1 \mu \mathrm{m}$ and $10 \times 10 \mu \mathrm{m}$ ) was performed in ambient air. Due to space limitations, only the $1 \times 1 \mu \mathrm{m}$ area scans are presented here.

\section{MPD of NHA on anodized Ti}

To combine the aforementioned NHA synthesis and anodized nanotubular Ti samples, MPD processing was used (Figure 1B). The MPD process was originally developed by Chameleon Scientific (Longmont, CO, USA) and enables the deposition of HA coatings onto metals, polymers, or other ceramics at thickness levels ranging from nanometers to tens of microns. The MPD process utilizes a corona discharge at a high voltage to allow coatings to be applied uniformly at low temperatures onto a wide range of substrates.
For this, the anodized Ti samples were preheated to $100^{\circ} \mathrm{C}$ for 1 hour. First, an 18 gauge needle was placed near the MPD opening while the substrate holder was attached on the inside of the vacuum chamber. The HA solution held in a reservoir was then added to the metallic needle. The solution was then dispensed at $2 \mathrm{~mL} / \mathrm{min}$ through the needle while 20 kV (SR6; Spellman Power Supply, USA) was applied. Electrical stress was induced by the applied voltage at the needle tip, resulting in corona discharge. The HA containing solution was ionized via this corona discharge, and HA was then deposited on the anodized nanotubular Ti by introducing the ionized solution into the vacuum chamber. After venting and substrate removal, the NHA-coated Ti substrates were thoroughly washed with deionized water to remove the unattached HA in solution. After the coatings, the samples were 

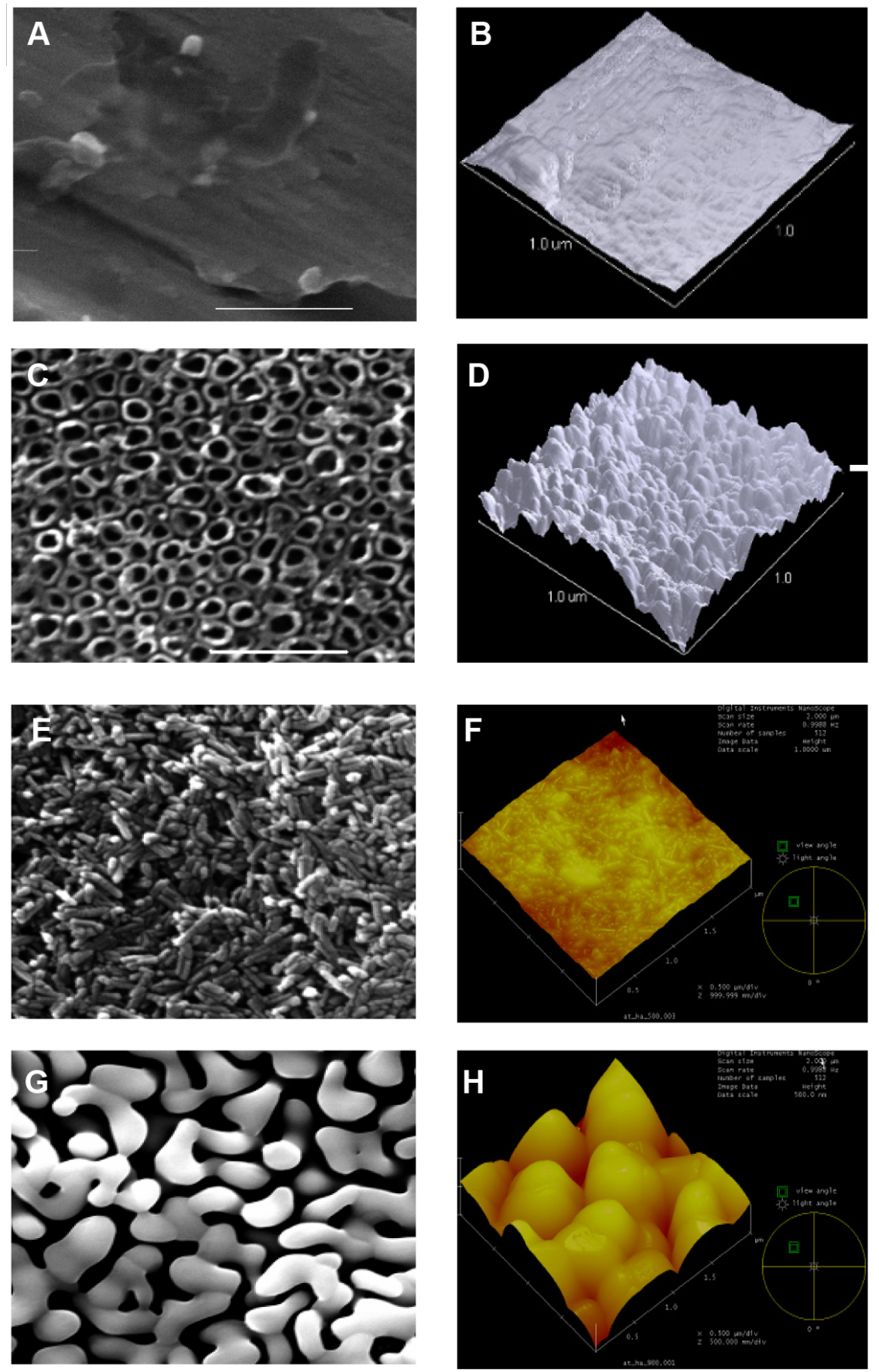

Figure 2 SEM and AFM surface morphologies of MPD-coated HA materials.

Notes: Plain Ti (A and B), anodized Ti (C and D), NHA-coated Ti (E and F) and MHA-coated Ti (G and $\mathbf{H})$. Scale bar $=\mathbf{I} \mu \mathrm{m}$ for all SEM and AFM pictures. Abbreviations: AFM, atomic force microscopy; HA, hydroxyapatite; MHA, micron sized hydroxyapatite; MPD, molecular plasma deposition; NHA, nanocrystalline hydroxyapatite; SEM, scanning electron microscopy; Ti, titanium.

removed from the deposition chamber and were allowed to cure at $500^{\circ} \mathrm{C}$ and $900^{\circ} \mathrm{C}$ for 2 hours. Lastly, the samples were kept in a dessicator until further use.

\section{Coating characterization}

The coatings were characterized by XRD, SEM, and AFM as described. AFM (Multimode SPM; Digital Instruments) was used to quantify surface roughness and surface area for all of the coatings of interest. AFM scanning was conducted at five random places, at a scanning rate of $1 \mathrm{~Hz}$, in tapping mode.

\section{Coating stability in cell culture media}

Coated substrates were placed in a shaking water bath at $37^{\circ} \mathrm{C}$ in a sealed container with Dulbecco's Modified 
Eagle's Medium (DMEM; Gibco) cell culture media supplemented with 10\% fetal bovine serum (Hyclone) and $1 \%$ penicillin-streptomycin (Gibco) in order to determine their stability. After 1 and 3 days of immersion, the samples were removed and thoroughly rinsed with distilled water, dried in a vacuum oven at $70^{\circ} \mathrm{C}$ overnight, and inspected using SEM.

\section{Osteoblast adhesion and morphology}

Ultraviolet light exposure $(250 \mathrm{~nm})$ for 4 hours was used to sterilize all substrates $\left(1 \mathrm{~cm}^{2}\right)$ prior to cell experiments. Human osteoblasts (bone-forming cells) (CRL-11372, population numbers 5-7; American Type Culture Collection) in DMEM supplemented with 10\% fetal bovine serum and 1\% penicillin-streptomycin (Hyclone) were seeded at a density of 3,500 cells $/ \mathrm{cm}^{2}$ onto the substrates of interest, which were then placed in standard cell culture conditions (humidified, $5 \% \mathrm{CO}_{2} / 95 \%$ air environment) for 4 hours. After the prescribed time period, substrates were rinsed in phosphate buffered saline to remove any nonadherent cells. The remaining cells were fixed with formaldehyde (Sigma-Aldrich), stained with Hoechst 33258 dye (Sigma-Aldrich), and counted (using five random fields per substrate) under a fluorescence microscope (Leica DM IRB). Experiments were run three times, and data were analyzed using standard Student's $t$-tests to determine statistical significance between means.

Osteoblast morphology was determined on the substrates after culturing cells for 4 hours and removing nonadherent cells by rinsing the substrates two times in phosphate buffered saline. To fix the cells, samples were soaked in 3\% glutaraldehyde (Sigma-Aldrich) for 60 minutes. Then, the substrates were placed in 1\% osmium tetroxide (Sigma-Aldrich) in $0.1 \mathrm{M}$ sodium cacodylate for 60 minutes. To prepare for SEM analysis, substrates were soaked in $0.1 \mathrm{M}$ sodium cacodylate, and the cells were then dehydrated by soaking in increasing $(35 \%, 50 \%, 70 \%, 95 \%$, and $100 \%)$ concentrations of anhydrous ethanol at 10 minutes each. The substrates were then placed in an ethanol/hexamethyldisilazane (SigmaAldrich) solution (1:1) two times for 10 minutes and were subsequently soaked in hexamethyldisilazane for 10 minutes and air-dried for 8 hours in a fume hood. Then, cells were visualized using SEM.

\section{Result}

\section{Material characterization}

Results of the present study confirmed the expected chemistry and nanocrystallinity of the synthesized powders (Figure 1A). Specifically, the NHA particles prepared here were rod-shaped: 40-100 nm long and 20-30 nm wide (diameter). Results also showed that the NHA Ca/P ratio in the nanoparticles was 1.63, slightly below the stoichiometric ratio of 1.67. Further, BET results from this study demonstrated large differences in particle surface area and sizes; specifically, micron sized hydroxyapatite (MHA) were found to be $600-800 \mathrm{~nm}$ while NHA were found to be $31 \mathrm{~nm}$ (both in diameter), with specific surface areas of approximately $8 \mathrm{~m}^{2} / \mathrm{g}$ and $160 \mathrm{~m}^{2} / \mathrm{g}$, respectively.

Prior to the MPD process, it was necessary to anodize Ti to increase surface area and available space for the NHA to deposit to increase bonding strength. As expected, the unmodified control Ti had surface features in the micron range (Figures $2 \mathrm{~A}$ and $\mathrm{B}$ ). However, after anodization, the Ti surface possessed numerous nanotubular structures uniformly distributed over the surface (Figures 2C and D). The nanotubular Ti surface inner diameters ranged from 70 to $80 \mathrm{~nm}$. As expected, due to the presence of the Ti nanotubular structures, AFM data proved that the untreated Ti surface was less rough $(10 \mathrm{~nm})$ than the anodized Ti (24 nm).

Using MPD, both SEM and AFM confirmed a homogeneous HA coating on Ti (Figures 2E-H). After coating, NHA particles were less than $30 \mathrm{~nm}$ wide and 100-120 nm long. In contrast, after coating and sintering, MHA particles were less than 50-100 $\mathrm{nm}$ wide and 600-800 nm long, possessing a nonuniform morphology. A closer examination showed a somewhat rough morphology at the nanoscale. Lastly, these characterization studies demonstrated that both of the coatings adhered well onto Ti as evident by the lack of cracks. Even after 24 and 72 hours of incubation in cell culture media, no cracks were found for any of the substrates (Figure 3 ). Interestingly, at both time points, the nanofeatures on the NHA coatings were still pronounced.

XRD data showed that after heat treatment (for 2 hours at $500^{\circ} \mathrm{C}$ ), single crystalline apatite structures were formed on the MPD-coated Ti surfaces (Figure 4). This is in contrast to the as-deposited coating (not heated), which was amorphous, as expected (data not shown). At $900^{\circ} \mathrm{C}$, both the HA and Ti crystallized and displayed phase transformations compared to the NHA coatings. Of special note was that only apatite and titanium dioxide $\left(\mathrm{TiO}_{2}\right)$ phases developed without the formation of other phases at $500^{\circ} \mathrm{C}$, suggesting the stability of both apatite and $\mathrm{TiO}_{2}$ structures in the NHAcomposite coatings when using the MPD method. This is in contrast to $\mathrm{HA}+\mathrm{TiO}_{2}$-composite coatings obtained by other methods: tricalcium phosphate, calcium Ti oxides, and calcium oxide, normally observed through other 

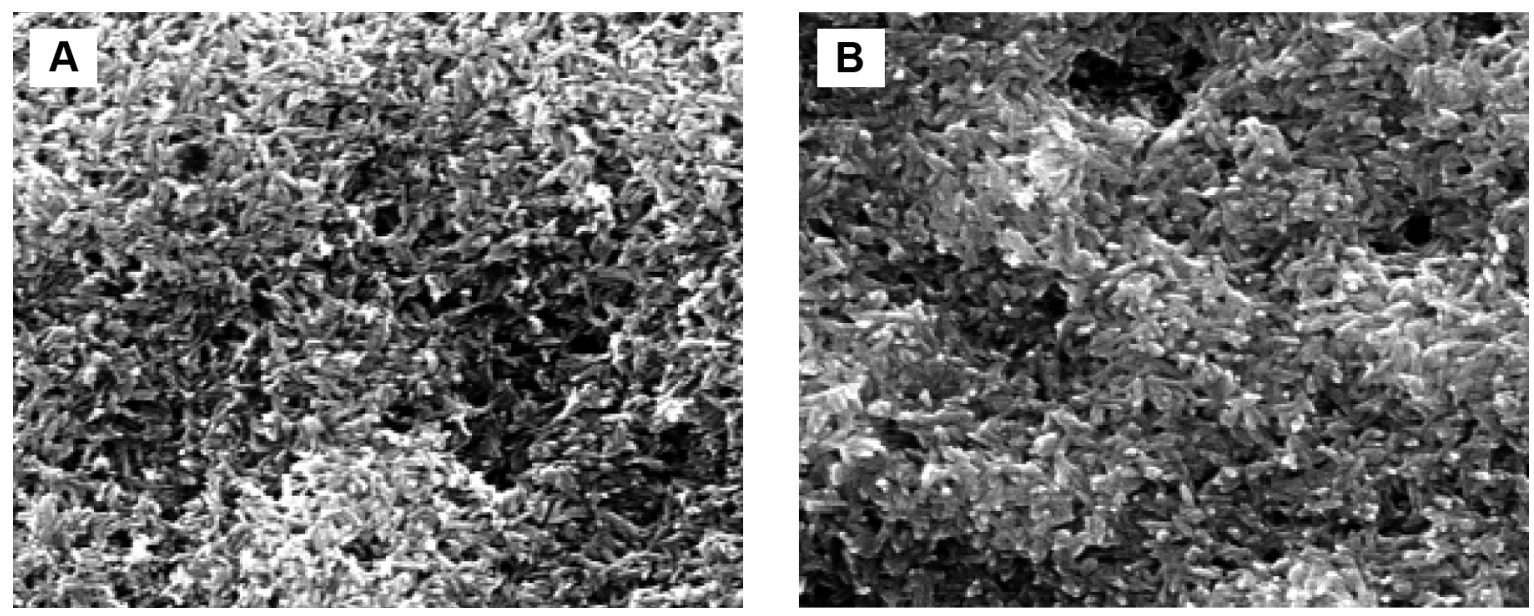

Figure 3 MPD coating stability in DMEM media.

Notes: SEM of NHA-coated anodized Ti after I day $(\mathbf{A})$ and 3 days (B).

Abbreviations: DMEM, Dulbecco's Modified Eagle's Medium; MPD, molecular plasma deposition; NHA, nanocrystalline hydroxyapatite; SEM, scanning electron microscopy; Ti, titanium.

coating processes, such as traditional plasma deposition, which clearly influences cellular responses, degradation, and coating stability.

\section{Osteoblast density and morphology}

Results of the present study provide the first qualitative evidence of increased osteoblast density on NHA-coated substrates compared with plain and anodized Ti (Figure 5). As supported in other studies, ${ }^{29-31}$ after 4 hours, quantitative osteoblast density increased on anodized nanotubular $\mathrm{Ti}$ compared to untreated Ti. However, for the first time, it is reported here that osteoblasts adhered in greater numbers on anodized nanotubular Ti coated with NHA compared to being coated with MHA and untreated Ti substrates. Interestingly, this study demonstrated that when coating Ti with MHA, osteoblast densities were less than those on the anodized Ti. Although requiring more investigation, such results suggest that one may not need to coat Ti with HA to improve a biological response, but rather simply anodize it instead. After 7 days of culture, similar trends were obtained.

As expected, for plain Ti, osteoblasts possessed a condensed morphology approximately averaging $45 \mu \mathrm{m}^{2} / \mathrm{cell}$. In contrast, osteoblasts were well spread on anodized $\mathrm{Ti}$ (averaging $110 \mu \mathrm{m}^{2} / \mathrm{cell}$ ), with numerous filopodia around the periphery of the osteoblast. Moreover, osteoblasts continued to be well spread on the NHA-coated anodized Ti, whereas MHA-coated nanotubular Ti surfaces showed poor cell features (Figure 5). These results are promising, as in order for osteoblasts to secrete a mineralized extracellular matrix, they must first adhere and be well spread on an implant surface.
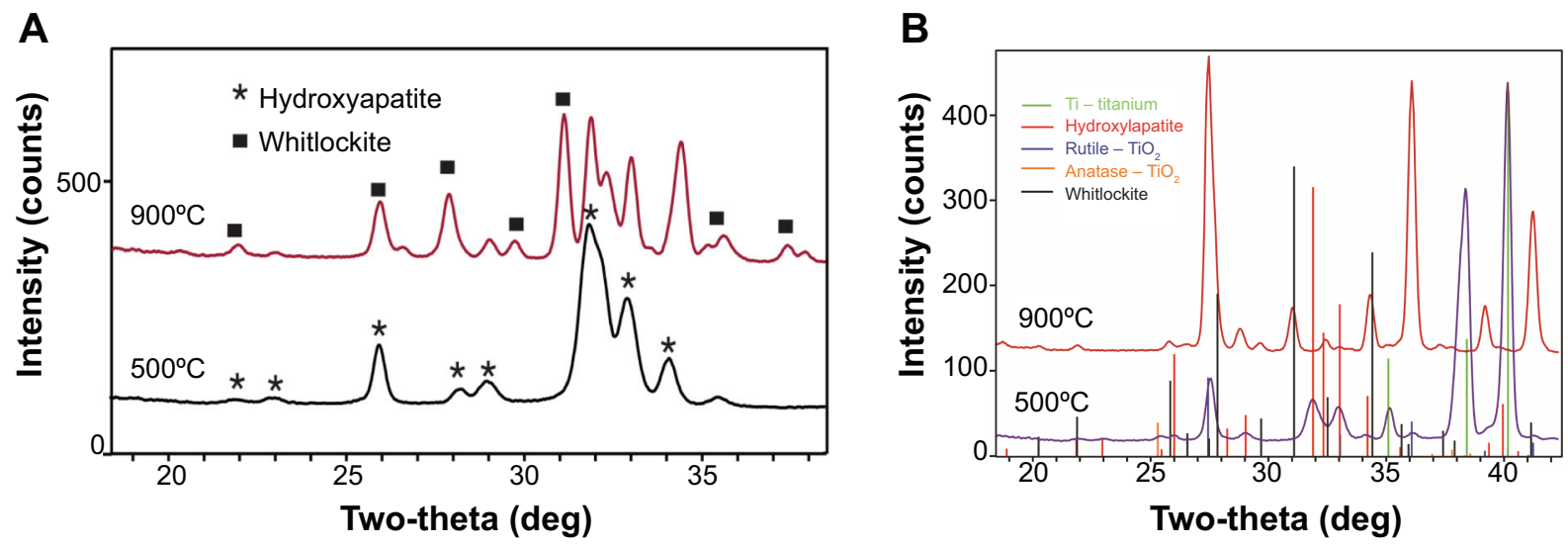

Figure 4 XRD spectra: NHA powder and MHA powder (A) and NHA-coated anodized Ti and MHA-coated anodized Ti (B). Note: The different phases of $\mathrm{TiO}_{2}$ or calcium phosphates are given in the figure.

Abbreviations: MHA, micron sized hydroxyapatite; NHA, nanocrystalline hydroxyapatite; Ti, titanium; XRD, X-ray diffraction. 

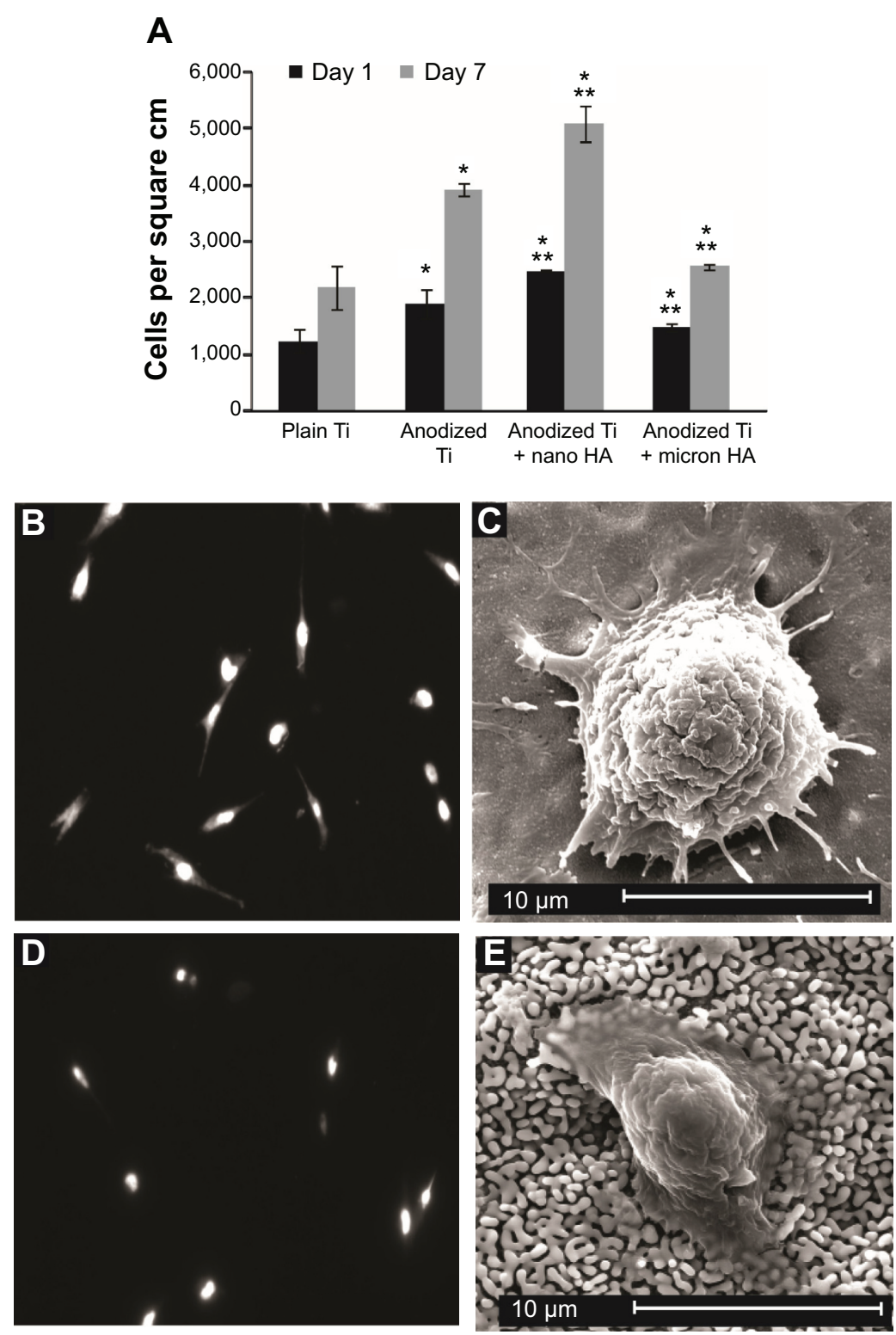

Figure 5 Increased osteoblast density on MPD-coated anodized Ti after I and 7 days.

Notes: Data are the mean \pm standard error of the mean; $n=3 ; * P<0.01$ (compared to plain $\mathrm{Ti}$ at the same time period); $* * P<0.0 \mathrm{I}$ (compared to anodized Ti at the same time period) (A). All 7-day densities were greater than I day samples, respectively. Qualitative fluorescence (B and $\mathbf{D})$ and SEM (C and $\mathbf{E})$ morphology of osteoblasts on NHA-coated Ti and MHA-coated Ti, respectively.

Abbreviations: HA, hydroxyapatite; MHA, micron sized hydroxyapatite; MPD, molecular plasma deposition; NHA, nanocrystalline hydroxyapatite; SEM, scanning electron microscopy; Ti, titanium.

\section{Discussion}

Results of this study showed improved osteoblast density after coating anodized nanotubular Ti with HA, demonstrating that the MPD process is a quick and effective process for the deposition of numerous materials on orthopedic implant materials. MPD is versatile, and numerous materials from metals to ceramics to polymers can be coated. It has become clear that most of these substrates are difficult to coat with nanomaterials while keeping bioactive nanofeatures, but it now may be possible using MPD, as this study shows.
As mentioned, MPD uses ionized plasma, which means when individual molecules are ionized at high voltage levels, they dissociate and may lose functional properties. It is therefore critical to evaluate biological activity of MPD-coated materials after the coating process.

XRD provided the first evidence that Ti coated with HA maintained their original HA diffraction patterns, identical to the HA powder prior to the MPD coating process. This confirmed that the crystallographic structure of HA was not significantly changed during the coating fabrication process. 
This is in contrast to HA coated on Ti using conventional plasma spray processes (which alters XRD HA spectra).

Of course, an important test of the retention of functional properties is an in vitro cellular density assay. Results of these cellular experiments provided additional evidence of the retention of the greater functionality of nanoscale HA towards increasing osteoblast density. Although future studies will focus on the long-term functions of osteoblasts (such as mineralization and alkaline phosphatase activity), osteoblast adhesion is a necessary prerequisite for such functions.

Nonetheless, this study provided critical evidence that MPD is a process worth further study as it is low cost, fast, robust, simple, and can retain attractive nanoscale properties for improving osteoblast densities. With the advance of nanomaterials and biotechnology, new orthopedic and dental materials can mimic the mechanical properties, surface topography, and chemical characteristics of bone through the application of the MPD process. Although future studies will have to delineate the exact mechanisms of greater osteoblast adhesion and spreading on nanoscale HA compared to MHA-coated anodized $\mathrm{Ti}$, our previous results on other materials hint at a plausible explanation. ${ }^{35}$ For example, our previous studies on noncoated NHA compared to MHA demonstrated a significant increase in surface energy and, thus, adsorption of vitronectin on NHA, which led to greater osteoblast functions. This can be easily understood by considering the significantly greater surface area that is present for NHA compared to MHA which can lead to increased exposures of the HA hydrophilic chemistry, and in turn, an overall greater hydrophilicity to adsorb hydrophilic proteins important for mediating osteoblast adhesion. This same mechanism has been elucidated by our group when determining why osteoblast functions are greater on anodized compared to unanodized $\mathrm{Ti}^{30}$

\section{Conclusion}

Here, it is shown that MPD is a novel process that can coat NHA on anodized Ti to increase osteoblast numbers compared with untreated Ti and MHA-coated Ti substrates. MPD is fast, inexpensive, and effective at retaining the novel biological properties of NHA. Although future studies will have to elucidate additional osteoblast functions and coating stability, this study demonstrates that MPD is a viable alternative process that should be further studied for a wide range of implant applications.

\section{Acknowledgment}

The authors would like to thank Tushar M Shimpi for assistance with some of the experiments.

\section{Disclosure}

The authors report no conflicts of interest in this work.

\section{References}

1. Li Z, Kawashita M. Current progress in inorganic artificial biomaterials. J Artif Organs. 2011;14(3):163-170.

2. Nomura N. Artificial organs: recent progress in metals and ceramics. $J$ Artif Organs. 2010;13(1):10-12.

3. Kokubo T, Kim HM, Kawashita M, Nakamura T. Bioactive metals: preparation and properties. J Mater Sci Mater Med. 2004;15(2):99-107.

4. Ramaswamy $\mathrm{Y}, \mathrm{Wu} C, \mathrm{Zreiqat} \mathrm{H}$. Orthopedic coating materials: considerations and applications. Expert Rev Med Devices. 2009;6(4):423-430.

5. Disegi JA. Titanium alloys for fracture fixation implants. Injury. 2000; 31(Suppl 4):14-17.

6. Guillemot F. Recent advances in the design of titanium alloys for orthopedic applications. Expert Rev Med Devices. 2005;2(6):741-748.

7. Long M, Rack HJ. Titanium alloys in total joint replacement - a materials science perspective. Biomaterials. 1998;19(18):1621-1639.

8. Webster TJ, Ejiofor JU. Increased osteoblast adhesion on nanophase metals: Ti, Ti6Al4V, and CoCrMo. Biomaterials. 2004;25(19):4731-4739.

9. Cook SD, Thomas KA, Kay JF, Jarcho M. Hydroxyapatite-coated titanium for orthopedic implant applications. Clin Orthop Relat Res. 1988;225-243.

10. Nakashima Y, Hayashi K, Inadome T, et al. Hydroxyapatite-coating on titanium arc sprayed titanium implants. J Biomed Mater Res. 1997; 35(3):287-298.

11. Lin A, Wang CJ, Kelly J, Gubbi P, Nishimura I. The role of titanium implant surface modification with hydroxyapatite nanoparticles in progressive early bone-implant fixation in vivo. Int J Oral Maxillofac Implants. 2009;24(5):808-816.

12. Yamada M, Ueno T, Tsukimura N, et al. Bone integration capability of nanopolymorphic crystalline hydroxyapatite coated on titanium implants. Int J Nanomedicine. 2012;7:859-873.

13. Jensen $T$, Jakobsen $T$, Baas $J$, et al. Hydroxyapatite nanoparticles in poly-D,L-lactic acid coatings on porous titanium implants conducts bone formation. J Biomed Mater Res A. 2010;95(3):665-672.

14. Hacking SA, Pauyo T, Lim L, Legoux JG, Bureau MN. Tissue response to the components of a hydroxyapatite-coated composite femoral implant. J Biomed Mater Res A. 2010;94(3):953-960.

15. Lee TM, Yang CY, Chang E, Tsai RS. Comparison of plasma-sprayed hydroxyapatite coatings and zirconia-reinforced hydroxyapatite composite coatings: in vivo study. J Biomed Mater Res A. 2004;71(4):652-660.

16. Ye X, Chen M, Yang M, Wei J, Liu D. In vitro corrosion resistance and cytocompatibility of nano-hydroxyapatite reinforced $\mathrm{Mg}-\mathrm{Zn}-\mathrm{Zr}$ composites. J Mater Sci Mater Med. 2010;21(4):1321-1328.

17. Duarte LT, Biaggio SR, Rocha-Filho RC, Bocchi N. Influence of hydroxyapatite on the corrosion resistance of the Ti-13Nb-13Zr alloy. J Mater Sci Mater Med. 2009;20(5):1009-1015.

18. Fathi MH, Salehi M, Saatchi A, Mortazavi V, Moosavi SB. In vitro corrosion behavior of bioceramic, metallic, and bioceramic-metallic coated stainless steel dental implants. Dent Mater. 2003;19(3):188-198.

19. Roy M, Bandyopadhyay A, Bose S. Induction plasma sprayed nano hydroxyapatite coatings on titanium for orthopaedic and dental implants. Surf Coat Technol. 2011;205(8-9):2785-2792.

20. Vidigal GM, Groisman M, de Sena LA, Soares Gde A. Surface characterization of dental implants coated with hydroxyapatite by plasma spray and biomimetic process. Implant Dent. 2009;18(4):353-361.

21. Han JY, Yu ZT, Zhou L. Hydroxyapatite/titania composite bioactivity coating processed by the sol-gel method. Biomed Mater. 2008;3(4): 044109.

22. Wang J, Chao Y, Wan Q, Zhu Z, Yu H. Fluoridated hydroxyapatite coatings on titanium obtained by electrochemical deposition. Acta Biomater. 2009;5(5):1798-1807.

23. Sandrini E, Giordano C, Busini V, Signorelli E, Cigada A. Apatite formation and cellular response of a novel bioactive titanium. J Mater Sci Mater Med. 2007;18(6):1225-1237. 
24. Hu R, Lin CJ, Shi HY. A novel ordered nano hydroxyapatite coating electrochemically deposited on titanium substrate. J Biomed Mater Res A. 2007;80(3):687-692.

25. Xia Z, Yu X, Wei M. Biomimetic collagen/apatite coating formation on Ti6Al4V substrates. J Biomed Mater Res B Appl Biomater. 2012;100(3): 871-881.

26. Sato M, Aslani A, Sambito MA, Kalkhoran NM, Slamovich EB, Webster TJ. Nanocrystalline hydroxyapatite/titania coatings on titanium improves osteoblast adhesion. J Biomed Mater Res A. 2008;84(1): 265-272.

27. Evis Z, Sato M, Webster TJ. Increased osteoblast adhesion on nanograined hydroxyapatite and partially stabilized zirconia composites. J Biomed Mater Res A. 2006;78(3):500-507.

28. Balasundaram G, Sato M, Webster TJ. Using hydroxyapatite nanoparticles and decreased crystallinity to promote osteoblast adhesion similar to functionalizing with RGD. Biomaterials. 2006;27(14):2798-2805.

29. Balasundaram G, Yao C, Webster TJ. TiO2 nanotubes functionalized with regions of bone morphogenetic protein-2 increases osteoblast adhesion. J Biomed Mater Res A. 2008;84(2):447-453.
30. Yao C, Webster TJ. Anodization: a promising nano-modification technique of titanium implants for orthopedic applications. $J$ Nanosci Nanotechnol. 2006;6(9-10):2682-2692.

31. Yao C, Slamovich EB, Webster TJ. Enhanced osteoblast functions on anodized titanium with nanotube-like structures. J Biomed Mater Res A. 2008;85(1):157-166.

32. Kim MH, Lee SY, Kim MJ, Kim SK, Heo SJ, Koak JY. Effect of biomimetic deposition on anodized titanium surfaces. J Dent Res. 2011; 90(6):711-716.

33. Das K, Bose S, Bandyopadhyay A. Surface modifications and cellmaterials interactions with anodized Ti. Acta Biomater. 2007;3(4): 573-585.

34. Rodriguez R, Kim K, Ong JL. In vitro osteoblast response to anodized titanium and anodized titanium followed by hydrothermal treatment. J Biomed Mater Res A. 2003;65(3):352-358.

35. Webster TJ, Ergun C, Doremus RH, Siegel RW, Bizios R. Specific proteins mediate enhanced osteoblast adhesion on nanophase ceramics. J Biomed Mater Res. 2000;51(3):475-483.
International Journal of Nanomedicine

\section{Publish your work in this journal}

The International Journal of Nanomedicine is an international, peerreviewed journal focusing on the application of nanotechnology in diagnostics, therapeutics, and drug delivery systems throughout the biomedical field. This journal is indexed on PubMed Central, MedLine, CAS, SciSearch $®$, Current Contents $\AA /$ Clinical Medicine,

\section{Dovepress}

Journal Citation Reports/Science Edition, EMBase, Scopus and the Elsevier Bibliographic databases. The manuscript management system is completely online and includes a very quick and fair peer-review system, which is all easy to use. Visit http://www.dovepress.com/ testimonials.php to read real quotes from published authors. 